of the separation of voids (in the observed b.c.c. void lattice) to the void diameter is smaller than the values actually observed. Stoneham's theory explains why such a lattice is stable once formed, but not how it is established in the first place.

Liou and his collaborators point out that the formation of a void lattice is analogous to the alignment of precipitate plates on different planes to form a preferred, stable array. This is very commonly observed in alloys formed by spinodal decomposition or by ordering, but had not hitherto been properly analysed. The theoretical basis of this phenomenon has now been rigorously treated by Perovic, Purdy and Brown (Acta Met. 27, 1075; 1979), who calculate the total elastic energy of a regular array of plate-shaped precipitates in a matrix, the precipitates lying on three mutually orthogonal planes. They show that such an array, subject to certain conditions (primarily a low interfacial energy and a large, elastically accommodated misfit between matrix and precipitate) can stabilise at a particular value of the separation of adjacent precipitates, so that further coarsening of the array is inhibited. This presumably is what happens in the formation of a void lattice, though in fact it is not yet known whether a fine void lattice forms first and then coarsens until it becomes stable, or whether the initially disordered voids move about in a kind of Brownian motion until the relative positions of a small group of voids happen to be correct to nucleate a lattice at its proper stable lattice parameter. In the case of a precipitate array, each precipitate plate creates an anisotropic. strain field around itself, and an isotropic medium is assumed, for simplicity; whereas in the case of voids, it is only the anisotropy of the medium which allows a void lattice to form at all.

Electron microscopy has shown that an array of precipitates can coarsen progressively by simultaneous dissolution of some portions of a precipitate plate and growth of other portions (Saunderson, Wilkes \& Lorimer Acta Met. 26, 1357; 1978 ), and voids or gas bubbles are known to migrate and merge by a similar mechanism. The growth of voids can be inhibited by small amounts of impurity, which may be fission products such as technetium; the impurity can gather at the void wall and prevent plating out of further vacancies into the void. Because of this, voids may even shrink in the later stages of irradiation (Evans $J$. Nucl. Mater. in the press). In such instances, the eventual shrinkage of the void population is in competition with the formation of a void lattice (which requires a minimum size of void to be attained). What is really required now is simultaneous ion-bombardment and electonmicroscopic observation of the same samples so that the stages of formation of a void lattice can be observed while they actually happen.

Most published photographs of void lattices show them to be distinctly defective (for instance, the photograph on page $\mathbf{3 3 8}$ of Liou's recent paper); the periodicity has a good deal of scatter. It would be interesting to know whether a void lattice, once formed, gradually loses such regularity as it possesses if it is progressively heated; just as a conventional superlattice in, say, $\mathrm{Cu}_{3} \mathrm{Au}$ gradually disorders as the alloy is heated. No experiments on annealing of void lattices seem to have been attempted, nor has anyone attempted to improve the regularity of a void lattice by very slow cooling (which is the method used to obtain highly perfect conventional superlattices). There are plainly a number of parallels between void lattices and conventional superlattices which deserve critical examination.

\section{A spherical structure for allophane}

\section{from Ian Smalley}

KosI Wada of Kyushu University has produced and collected evidence which strongly suggests that the clay mineral allophane, long regarded as an 'amorphous' constituent of soils, has a unique spherical structure. Although plates and tubes have already been encountered in clay mineralogy, this seems to be the only occurrence of spheres (Proc. Int. Clay Conf., eds Mortland \& Farmer, 537; Elsevier, New York, 1978). Taylor and Wilson (Clays and Clay Miner. 27, 261; 1979) describe allophane as consisting of an aluminosilicate core, about $5 \mathrm{~nm}$ in diameter, coated to a variable extent with amorphous aluminium hydroxide. The clay surface has hydroxyl and hydronium groups associated with the polymeric hydroxide and the aluminosilicate, and these (by their protonation and deprotonation) are responsible for the $p \mathrm{H}$ dependent particle charge developed in aqueous systems. (The nature and properties of variable-charge soils containing allophane will be discussed at the International Society of Soil Science meeting in New Zealand in 1981.)

Allophane usually coexists with imogolite, but the two can be distinguished easily by their shapes, spherical allophane contrasting with tubular imogolite. In 1971 Kitagawa first discovered by high resolution electron microscopy that allophane consists of spherical, hollow unit particles with an average diameter of 5.5 nm. Hemni and Wada (Am. Miner. 61, 379 ; 1976) observed that similar particles occurred in sixteen allophanic clays, irrespective of their ages and lithological

Ian Smalley is with the Soil Bureau, Department of Scientific and Industrial Research, Lower Hutt, New Zealand. compositions, or the origins of the volcanic ashes and pumices from which the clays were separated. They estimated the external diameter of the particles to range from 3.5 to $5.0 \mathrm{~nm}$ and the thickness of the wall to be $1.0 \mathrm{~nm}$ or less. The thickness of the wall is broadly consistent with that of a defect kaolin structure.

Wada and Wada (Clay Miner. 12, 289; 1977) determined the density of allophane by float-sink tests in $\mathrm{CH}_{3} \mathrm{OH}$-, $\mathrm{CH}_{3} \mathrm{COCH}_{3}-, \mathrm{C}_{6} \mathrm{H}_{6}-$ and $\mathrm{CCl}_{4}-\mathrm{C}_{2} \mathrm{H}_{2} \mathrm{Br}_{4}$ mixtures as $1.84,1.94-1.98,2.35$ and $2.35-2.39 \mathrm{~g} \mathrm{~cm}^{-3}$ respectively. Either water molecules only fill the voids of the spherule wall, or all liquid molecules (including $\mathrm{C}_{2} \mathrm{H}_{2} \mathrm{Br}_{4}$ ) occupy these spaces, the first possibility applying if the wall contains small pores and the second if it contains large pores. It is reasonable to assume that 'defects' in the silicate structure produce such pores or openings in the wall, and the passage of water molecules through these has been confirmed by the ease with which $\mathrm{OH}$ ions can be replaced by their deuteriumcontaining equivalent (OD') in allophane.

Wada concludes that the structural formulae for allophanes are in general consistent with the available electron optical and density evidence. The modification of the kaolin layer caused by the size and shape of the unit particles, and by the occurrence of defects, may account for several features of allophanes, especially the absence of both basal and two-dimensional X-ray reflections, the broadness and poor resolution of Si-O-(A1) absorption bands in the $1100-900 \mathrm{~cm}^{-1}$ region of the infrared spectra, and the remarkably continuous loss of water with increasing temperature due to dehydration and dehydroxylation. The observed electric charge and acid characteristics of allophanes are also broadly consistent with what is predicted from the structural formule.

\section{Soil biology and land use}

\section{from Clive A. Edwards}

A RECENT symposium* addressed the often neglected subject of the impact of human activities on the soil - the resource on which all plant and animal life depends. Although man's use of the land can often have damaging effects on the soil, work presented at the symposium showed that with care, such effects can be minimised, and careful manipulation of some of the multitide of soil organisms can benefit man by increasing soil fertility, and can help to reclaim polluted land and dispose of an increasing volume of human and animal waste.

There has been much discussion on how 